\title{
A Simplified Mobile Ad Hoc Network Structure for Helicopter Communication
}

\author{
Abdeldime Mohamed Salih Abdelgader, ${ }^{1,2}$ Lenan $\mathrm{Wu},{ }^{1}$ \\ and Mohammed Mohsen Mohammed Nasr ${ }^{1}$ \\ ${ }^{1}$ Southeast University, School of Information Science and Engineering, Nanjing 210096, China \\ ${ }^{2}$ Karary University, College of Engineering, Electrical and Computer Department, 12304 Khartoum, Sudan
}

Correspondence should be addressed to Abdeldime Mohamed Salih Abdelgader; abdeldime@hotmail.com

Received 10 October 2015; Revised 30 January 2016; Accepted 7 February 2016

Academic Editor: Mohamed Gad-el-Hak

Copyright (C) 2016 Abdeldime Mohamed Salih Abdelgader et al. This is an open access article distributed under the Creative Commons Attribution License, which permits unrestricted use, distribution, and reproduction in any medium, provided the original work is properly cited.

\begin{abstract}
There are a number of volunteer and statutory organizations who are capable of conducting an emergency response using helicopters. Rescue operations require a rapidly deployable high bandwidth network to coordinate necessary relief efforts between rescue teams on the ground and helicopters. Due to massive destruction and loss of services, ordinary communication infrastructures may collapse in these situations. Consequently, information exchange becomes one of the major challenges in these circumstances. Helicopters can be also employed for providing many services in rugged environments, military applications, and aerial photography. Ad hoc network can be used to provide alternative communication link between a set of helicopters, particularly in case of significant amount of data required to be shared. This paper addresses the ability of using ad hoc networks to support the communication between a set of helicopters. A simplified network structure model is presented and extensively discussed. Furthermore, a streamlined routing algorithm is proposed. Comprehensive simulations are conducted to evaluate the proposed routing algorithm.
\end{abstract}

\section{Introduction}

In the recent years, Ad hoc networks emerged with the rapid development of communication technologies. Typical ad hoc approaches consider nodes with similar capabilities that move in random patterns relative to one another over time and the communication is separated from the other activity of the node. This type of networks is known as mobile ad hoc network (MANET), which is a wireless network that is formed by a collection of self-organizing mobile nodes [1]. Each node communicates with its neighbors over a shared wireless medium. In MANET, wireless nodes cooperate to relay packets over multiple relay hops from source to destination that is known as multihop network [2-4]. Due to the lack of central management, nodes in MANET are designed to act as end system and routers for other nodes. The network is connected dynamically and does not rely on any preexisting network infrastructure. In MANET, nodes are free to move and have the capability to deliver messages in a decentralized manner. MANET has been utilized in vehicular environments to form the vehicular ad hoc networks (VANETs) $[3,5]$. VANETs recently emerged with great interest due to their impact in reducing traffic jams and increasing safety. It can also be used for providing many services to passengers, drivers, and authorities. VANETs are limited only to urban and highways areas and they are difficult to work in rugged environments and some extraordinary situations without special communication equipment and facilities.

Unlike planes, helicopters have the advantage of not requiring a runway for taking off or landing. Therefore, they can carry both people and goods to virtually impassable locations often not connected by road and only accessible on foot with difficulty. Helicopters are therefore indispensable tool for rescue organizations, armies, navies, and especially air forces. Recently, the ambulance service has a statutory responsibility to respond to traumatic and medical emergencies. Fire and rescue authority has a statutory duty to deal with fires, road traffic accidents, and maybe certain types of 
terrorist related incidents such as mass decontamination. Fire and rescue authorities with discretion require responding to events such as flooding or animal rescue [6]. Helicopters can be a valuable and versatile asset in any Search and Rescue (SAR) events $[4,7,8]$. Emergency services personnel are increasingly calling on the support of a helicopter air assistance [9]. Radio contact is still of great importance for communication between helicopters, planes, and ground stations. The operation environment is different from that of vehicular networks and traditional ad hoc networks. Helicopters outperform the performance of vehicles, particularly in cases that need fast response and in rugged or distant areas. However, it requires special and reliable communication requirements to provide reliable services. Helicopters network can also serve as alternative emergency communication system in case of natural disasters, when there is lack of ordinary ground communication systems. Furthermore, in many situations, communication between helicopters and ground vehicles or operation center is highly required.

Recently, the increased capabilities in embedded systems have enabled unmanned flight through the removal of the human pilot from the aircraft cockpit. As the applications of drone and Unmanned Aircraft System (UAS) expand, the principal issues of communication technologies are flexibility, adaptability, and controllability of the information/data flows. Future systems will be net-centric and rely on mobile ad hoc networking to provide real-time connectivity among cooperating agents and to provide backhaul of sensor and telemetry data from mobile nodes to a data repository or command center [10]. There is a current push towards the development of simple, small, low-cost UAS that work individually and in cooperative teams to accomplish complex and dangerous missions. These networks can be applied to numerous applications, including diffuse gas and plume detection [11], coordinated search [6] and reconnaissance [12], and in situ atmospheric sensing [13], as agents in the battlefield [14], aerial photography, and mass decontamination, and as components in command and control architectures. With smaller, cheaper UAS that have limited sensor capabilities, cooperative control relies heavily on communication with neighbors $[6,15-17]$. That means, by forming a multihop communication network, information can be efficiently shared among helicopters or UAS. Consequently, this will increase the overall mission capabilities and extend the operational range.

Communication networks between and through aerial vehicles are the backbone of the current battlefield communications. For instance, Google recently established a research and development project with the mission of offering Internet access to rural, rugged, and remote areas. The project is denoted as Project Loon [18]. The project uses high-altitude balloons placed in the stratosphere at an altitude of about $18 \mathrm{~km}$ to create an aerial wireless network with up to 4G-LTE speeds. Project Loon balloons float in the stratosphere, twice as high as airplanes and the weather. In the stratosphere, there are many layers of wind, and each layer of wind fluctuates in direction and speed. Loon balloons go where they are needed by rising or descending into a layer of wind blowing in the desired direction of travel. By associating with telecommunications companies to share cellular spectrum, loon balloons can enable people to connect to the balloon network directly from their phones and other LTE-enabled devices. The signal is then passed across the balloon network and back down to the global Internet on Earth. Each balloon can provide connectivity to a ground area (footprint) about $80 \mathrm{~km}$ in diameter using LTE wireless communications technology [19]. In a similar manner, helicopter network can provide Internet $\backslash$ network access for a specific area and for a particular mission by connecting ground users to a backbone network in a certain time.

Present helicopters communication systems use specialized high-cost radios that operate in designated military radio and other communication bands. Small low-cost Commercial Off-the-Shelf (COTS) radio equipment combined with powerful computer processing can be mounted on helicopters or small UA and has the potential to revolutionize battlefield communications and open up many scientific and commercial applications. One of COTS technologies is the IEEE 802.1x wireless LANs that connect wireless mobile nodes to a fixed infrastructure and is being widely deployed, including in UAS applications [15, 20]. Both IEEE 802.11 and IEEE 802.16 define Peer-to-Peer (P2P) and wireless ad hoc networks, where an end user communicates to users or servers on another local area network (LAN) or other users using its access point or base station. More interesting applications are possible when swarm of helicopters connect to each other in peer-to-peer ad hoc meshed wireless networks $[2,21]$. This can form directly or through multihop connected airplane, a new kind of MANET called aerial vehicular ad hoc networks (AVANETs).

The ability and performance of utilizing MANET as platform for connecting helicopters in one network have not been thoroughly investigated. MANET can be used in communicating a group of helicopters or drones working in specific mission to each other. It can also be used to communicate a helicopter or drone to the data center. Nowadays, people activities extended to deserts and other rugged environments for many purpose such as rescue, exploration, transportation, research, and mining. Helicopters can serve a lot in these areas. Improving the transmission of information about the accidents, weather, and locations in these areas will provide people with a higher degree of safety and comfort.

To the best of our knowledge, the use of ad hoc networks in helicopter communications has never been previously studied, despite their reliability, scalability, high data rate, bandwidth, diversity of application, and minimum cost compared to the existing solutions. This and the promising expected applications are the motivations of this work. This paper addresses the utilization of MANET in forming AVANET that consists of helicopters or UAS nodes. The main objective of this work is to investigate the utilization of MANET as alternative data communication link for helicopters, drones, or UAS environments. We present a simple model for the network structure and extensively discusse the different expected communication scenarios. In the proposed structure, a swarm of helicopters are clustered to one or multiple clusters which can communicate to some central 
servers through a wireless mesh backbone as well as Internet. Within each cluster, a self-organizing MANET is formed such that all helicopters are connected to all others within the same cluster. As the research on this topic, along with other related issues, is still in its infancy, we bring the attention to network structure formation and information routing. Accordingly, we propose a simple routing protocol. Furthermore, as many MANET routing protocols, such as Clustering Based Routing Protocol (CBRP) [22], use the ID number of the nodes to select the cluster head, this paper proposes a novel swarm head ( $\mathrm{SH}$ ) election approach which considers the communication equipment, velocity, and location of the helicopters in the election process. The proposed $\mathrm{SH}$ election approach uses a weighting mechanism to achieve a better utilization of all the nodes and perform optimum SH selection by using the election priority factor (EPF) of the nodes. The selection based on the location, equipment, and velocity of the nodes has never been addressed by other existing protocols which only use the ID or velocity to elect the SH.

The rest of this paper is organized as follows. Section 2 tackles ordinary helicopters' communication techniques and their limitations. This section also presents the promising communication scenarios which can be achieved by utilizing ad hoc networks for helicopter networks. In Section 3, we discuss the research opportunities which have not been considered by this work. The proposed network structure model and the $\mathrm{SH}$ election algorithm are presented in Section 4, while the routing algorithm is proposed in Section 5. Section 6 presents our simulations settings and their results. Section 7 concludes this work.

\section{Helicopter Communications}

Ordinarily, communications between air ambulance, police air support, SAR helicopter, and land SAR teams usually are via the ground to air channel as identified in the National Band Plan which is VHF High Band Land Search and Rescue Radio Channels (VHF-HBLS-RRC) [9]. Communications with coastguard rescue teams uses VHF channel $156 \mathrm{MHz}$. Communication between responding helicopters usually uses Scene of Search 123.1 MHz AM. SAR and some air ambulance helicopters are often fitted with VHF/UHF/HF and FM radios [9]. Some helicopters carry the Airwave Communications System (ACS), providing Terrestrial Trunked Radio (TETRA) communications used by emergency services and police through several talk groups [9]. In addition, the ACS provides a means to make telephone calls from the helicopter to mobile or PSTN telephone numbers. Some helicopters also carry a spare FM radio that can be deployed to incident control or to mobile ground units. Some kinds of helicopters, such as the Maritime Coastguard Agency (MCA) helicopters, have a satellite link (Satcom) and there is potential to communicate directly by phone [23]. Specific aspects of operations, such as hovering, winching, or refueling, may make it difficult to contact or get a reply from the crews. Although crew can monitor all radios throughout the copilot, these systems may not function with high efficiency, in case of urgent messages and life-threatening situations. Terrain masking can also be a factor and, in this case, emergency services may consider using local MCA units or MRTs helicopters (Mountain Rescue Teams) to enhance communications using satellite links. Emergency situations sometimes necessitate exchange of some information such as images, maps, and video that require high data rate and bandwidth. Besides, in some special missions an immediate exchange of information between helicopters themselves is quite needed. Conventional VHF/UHF communication systems are not the efficient way to carry this type of information.

Nowadays, Aircraft Communications Addressing and Reporting networks (ACARs) are under operation in the aeronautical world for aircraft-ground communications to offer a cockpit/maintenance/cabin operation. The ACARs data link service uses VHF radio channels. The VHF ground stations coverage is "line-of-sight." This means that a station at an airport can provide coverage to aircrafts located at the airport and above the airport within a cone limited by the curvature of the earth.

ACARs usually share the VHF band with voice communications, because most channels had been assigned to voice when ACARs was implemented. Thus, there were not enough VHF channels to assign a dedicated frequency to each ACARs ground station. This required the ACARs VHF design to allow service providers to have all their ground stations across a region use the same base frequency. Furthermore, by using the traditional VHF voice systems, the data rate in ACARs system is limited to only 2.4 kilobits per second. At a higher data rate, the rate of errors in decoding the received signal would rise and communications would become impossible. Moreover, there are already no VHF frequency channels available for allocation to new VHF ACARs services in Europe. This will soon be true in certain parts of USA. Therefore, to increase VHF data link capacity further, it will be quite essential to implement a system providing more capacity per channel. Although some helicopters have satellite communication or ACS Links, these solutions have limited bandwidth, are very expensive, and are only used for necessary voice calls [23]. For instance, in surveillance, monitoring, or rescue operations, the image or video of the target area must be relayed from the helicopter to the control center with a very strict delay bound, and it requires high bandwidth. In addition, by the help of the technological advancements on sensor technologies, it is possible to collect data with very high resolution, and this makes the bandwidth requirement much higher. The collaboration and coordination of multiple helicopters also need additional bandwidth resources. Sometimes, a type of communication to provide comfortable applications, such as entertainments, Internet services, and others, for the working team is also needed. In these cases, ad hoc network possibly is one of the alternative cheap solutions.

Ad hoc networks can be used to handle the communications between helicopter nodes using different scenarios:

(1) They can be used as internal communication between helicopters. Using this scenario a group of helicopters, drones, UA, or aircrafts can form together a full or half mesh topology LAN by which they can communicate to each other as shown in Figure 1(a). Forming this 


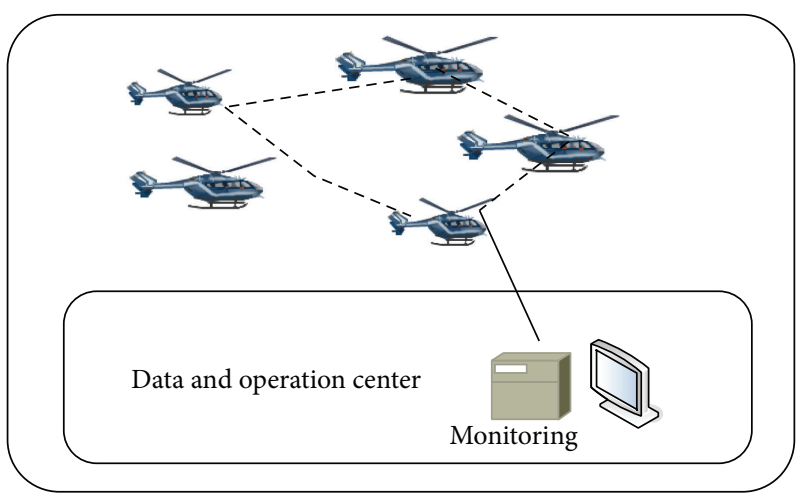

(a)

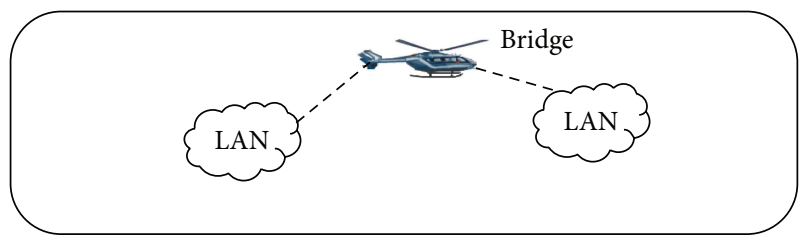

(b)

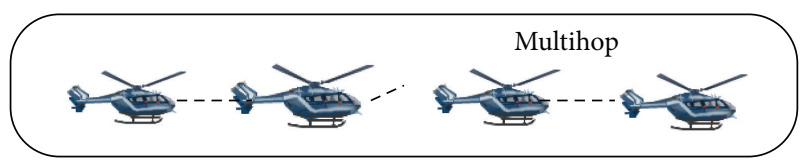

(c)

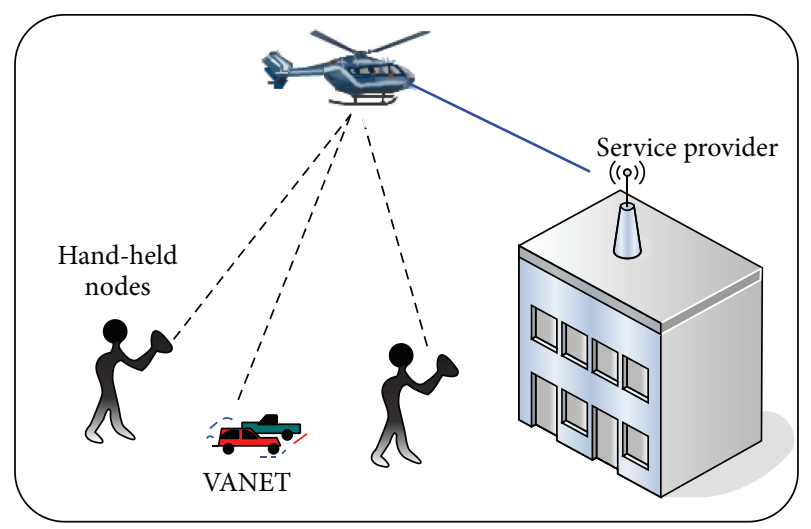

(d)

FIGURE 1: Helicopter network scenarios. (a) LAN and WAN. (b) Helicopter as bridge. (c) Extending network coverage range through multihops. (d) Providing Internet/network access for some users in specific area.

network can enable them to share and exchange resources and information between each other.

(2) They can be usedas a bridge in case of natural disasters or in special scenarios in which a communication between two networks is needed when the ordinary communication systems are lacking. This can be done using a rescue helicopter or small UA, to connect two nodes or networks as shown in Figure 1(b). This scenario can be temporarily implemented for a particular mission, when there is some natural obstacles which prevent installing ordinary network infrastructure, such as in seas (to connect two ships), across rivers, and over mountains.

(3) They can be used to extend the communication range: The range of the network can be extended beyond the ordinary distance of $\mathrm{Wi}-\mathrm{Fi}$ or any other wireless technology, using the ad hoc multihop characteristic, as shown in Figure 1(c). This scenario has many applications using helicopter nodes. It can be used to provide temporary link to certain location in some circumstances. This characteristic is very useful in case of natural obstacles, natural disasters, or loss of ordinary communication infrastructure in specific location. The environment also affects the communication coverage of the ordinary VANETs. Obstacles, such as mountains, walls, or buildings and constructions, may block the radio signals. AVANET can help to operate behind the obstacles, and it can extend the scalability of multihelicopter applications [24].

(4) They can be used to connect to a central point: A group of helicopters or air crafts can be connected to form a local area network (LAN) and then select one of them to act as gateway to connect to the central operation network. That means that they can exchange and share resources and information with the operation center using this topology, as shown in Figure 1(a).

(5) Helicopter or drone networks can be an active part of the upcoming technologies Internet of Vehicle (IoV) and Internet of Things (IoT). Providing Internet connection and network access to helicopters would provide a promising applications for many activities such as that of Figure 1(d).

(6) Helicopter networks can also serve as a large-scale wireless sensor network (WSN) for future ITS and many other systems, particularly in natural disasters situations and rugged environments, because every helicopter can be regarded as a super sensor node. Helicopters are commonly equipped with thermometers, microphones, one or more cameras, ultrasound radar, GPS, and other sensory features. A WSN of such capabilities is unprecedented and can extend perceptive computer systems to every point of the world. That means that, without the need for restrictive infrastructure, information can be shared in a peer-to-peer manner.

\section{Important Consideration}

The use of MANET in helicopters and drones networks is a new wide field of research. Up to our knowledge, this work seems to be the first one that studies the ability of using MANET to provide communication between helicopters. Therefore, many works require to be conducted in order to provide a complete investigation and implementation of MANET in such kind of environments. Many researches are required to consider the physical, MAC, and application 
layers in more details and study the suitability of the existing MANET and VANET solutions for AVANETs. Considering that helicopters can be a part of intelligent transport system (ITS), the use of wireless access in vehicular environment (WAVE) standard is also need to be investigated, as this standard is particularly designed for vehicular networks, and some AVANETs' characteristics may not be similar to that of VANET. Moreover, security is one of the most important issues, as this paper does not include a security solution for communication between helicopters in different scenarios. The existing solutions need to be investigated to ensure their suitability, reliability, latency, and scalability for these types of networks.

Furthermore, a general model for modeling the speed and the distribution of helicopters is much needed. Many other investigations and studies, which are related to helicopter network applications, routing, MAC, and physical layers can also be conducted. The implementation of OSI model in such type of networks may not fit well due to some constraints imposed by the nodes itself and the environment. Therefore, cross layering techniques are much needed to facilitate the interaction between layers and make it possible to tune layer parameters so that the overall performance can be improved. Many MAC and routing protocols [21, 25-27] can be used in this type of network but their impact, performance, reliability, and suitability need to be intensively examined. Additionally, the effect of these communication systems on the safety of the helicopter is one of the most important issues that is required to be carefully studied. There are many challenges for helicopter networks such as modeling mobility, connectivity, broadcast method, and routing protocols. The above stated points and many others are the next phase of research in AVANET. This paper proposes a general structure for the helicopters network and presents a routing mechanism for information exchange. The communication problem initially can be solved by using other systems' technologies, or by utilizing other researches' results $[10,15$, $23,28]$, but the high mobility and the dynamic change in topology and environments remain the biggest challenges, which require special attention. Hence, what set this work apart are the exploration and the use of MANET technologies and their induction into the scope of the proposed structure model, although not all technical difficulties are completely eliminated.

\section{The Proposed Network Model}

The network scenarios adopted throughout this paper are rescue environments or a group of helicopters/drones working in specific mission. The network structure of the proposed model is shown in Figure 2. We assume a number of helicopters $(N)$. The maximum numbers are not more than $N_{\max }$. Each of these helicopters is moving with velocity equal to $v_{I}$. When the number of nodes in one group exceeds $N_{\max }$, it will be divided into $g=K / N_{\max }$ neighbor groups; $K>$ $N_{\text {max }}$ is the total number of helicopters. In this paper, we use the notation swarm to represent a group of $N$ helicopters moving in the same direction and within a communication coverage range. The basic coverage range $(L)$ between two

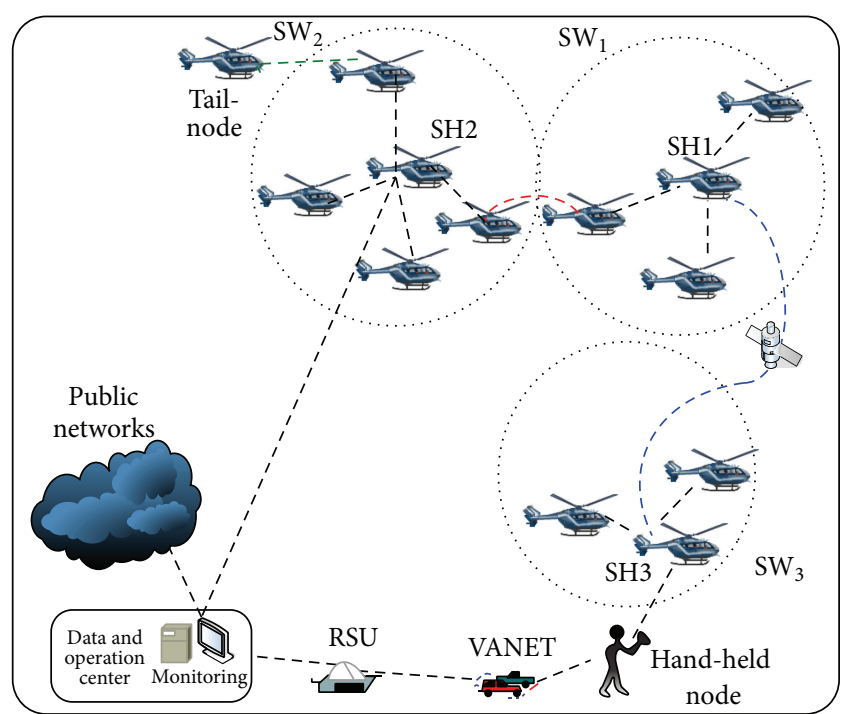

FIGURE 2: Simple structure for helicopters communication network.

helicopter nodes $(I, J)$ is according to (1), where $x, y, z$ are the coordinates of nodes. The presence of multihop operation can extend the coverage range to $L_{\max }$. The multihop operation can be conducted between at least three helicopters. The maximum distance between the first and the third one does not exceed $2 \times L$. That means that the maximum coverage distance in one swarm can be up to $L_{\max } \leq(N-1) \times L$. The maximum value of $L$ depends on the type of the physical layer communication technology, such as IEEE 802.11p and IEEE 802.16. Conisder

$$
L \leq \sqrt{\left(x_{i}-x_{j}\right)^{2}+\left(y_{i}-y_{j}\right)^{2}+\left(z_{i}-z_{j}\right)^{2}} .
$$

The mission of the swarm can be rescue operation, aerial reconnaissance, or military mission as well as alternative emergency communication system in case of natural disasters when there is lack of ordinary communication systems. The communication conditions can be according to three scenarios shown in Figure 2. The first scenario is the communication among the swarm members. The second one is the communication between two or more swarms, in case of very large number of helicopter nodes or when two or more swarms are moving in different directions. The third scenario is the communication between the swarm members and the ground operation center, the ground VANET nodes, or the communication to ground hand-held node which is a soldier or a victim that requires rescue. Practically, the number of helicopters moving in one direction for specific mission usually is very small. The small number of nodes eases swarm management and provides good performance. However, in some cases the large number of nodes may offer more alternative communication routes and facilities.

In this model, we assume that all helicopters are equipped with ad hoc radio equipment in addition to the traditional VHF/UHF systems used for voice communications. The ad hoc uses the specification of IEEE 802.11p to form an ad hoc network with a coverage range about $1 \mathrm{Km}$. Using 
802.11 with high-gain antenna can extend the coverage up to $20 \mathrm{~km}$; however, this requires more power, while the utilization of IEEE 802.16 can increase the coverage to more than $40 \mathrm{~km}$. However, some nodes may have other types of direct communication links. Therefore, this paper classifies helicopters according to their equipment into two types. The first type is that equipped with satellite link or any direct data communication link to ground terminals. We will use the notation $S_{1}$ to represent this type. The second type is the helicopters equipped with ad hoc network onboard units in addition to the ordinary voice communication links. We use the notation $S_{2}$ to represent this type of helicopters.

4.1. Swarm Formation. Helicopter/drone initiates the communication by broadcasting a HELLO message to discover the surrounding nodes. The HELLO message contains the information of the helicopter. These pieces of information include the ID number, velocity, and location and direction in degree and communication equipment type. We define a transitional state called $S_{-} U N D E C I D E D$ for smoother operation of swarm formation. "Undecided" means that a node is still in search for its host swarm. Other nodes within the coverage region upon receiving the message will respond by sending a REPLY message. In case of the responding node already joining an existing swarm, REPLY message will include its ID number, velocity, location, direction, and the ID number of the swarm. If it is not a member of a swarm it will send FREE message that includes its direction, ID number, velocity, and location. In case of the fact that the responding node is the $\mathrm{SH}$ of an existing swarm, it will send its information and the swarm information which include the amount of current nodes $(N)$ in the swarm. If $N<N_{\max }$, the $\mathrm{SH}$ will include an invitation message (INVITE) for the Undecided helicopter to join the swarm. The helicopter will directly become a swarm member and has all the information of the swarm and it can use the swarm facilities. It will change its state to "S_member." The direction is one of the important factors that qualify any helicopter to join a certain swarm. The direction should be not more than $\theta$ degree of the $\mathrm{SH}$ direction.

If $N \geq N_{\max }, \mathrm{SH}$ will send to the new node a JOIN GUEST message. The JOIN_GUEST msg includes the information of the nearest neighbor node to the guest one. The $\mathrm{SH}$ finds the nearest nodes using the information of its swarm and the location of the guest node. The new node will form a temporary network with the nearest neighbor node. The nearest node will work temporarily as relay node to carry the information of the guest node through SH to the rest of the network. The Undecided node will be treated as Tail-Node (TN) of the nearest node as shown in Figure 2. This situation will continue until the Undecided helicopter joins a swarm. In case of the responding node of type Undecided, the two nodes will store the information and directly start the $\mathrm{SH}$ election process according to the $\mathrm{SH}$ election procedure described in Section 4.2. After formation of the new swarm, the nearest node and its TN will act as relay between the two swarms. The swarm formation is not limited to the nodes that located within one MANET coverage; a set of nodes moving in the same direction can form a swarm using multihop operation until the number of nodes equals $N_{\max }$.

As a rule, $\mathrm{SH}$ and the neighbor $\mathrm{SH}$ can communicate directly if they are in the same ad hoc coverage. Nonetheless, in the actual situation of the physical structure of the network, SH1 and its neighbor SH2 may not necessarily to be within the same ad hoc communication range. Therefore, SH selects boundary members to act as a relay between the neighbor SHs and forward information. Therefore, swarm formation not only is the process of building the swarm structure and electing the $\mathrm{SH}$, but also includes the operation of determining the boundary nodes used to communicate between the adjacent swarms as shown in Figure 2.

4.2. Swarm Head Election. $\mathrm{SH}$ is responsible for route finding and organizing of interswarm communication. It is also responsible for finding the best route to distant destinations by exchanging its swarm information with other SHs. In many situations, $\mathrm{SH}$ participates in information forwarding process. Therefore, helicopter with best resources and facilities, located in a suitable location and having an average velocity among other nodes, is qualified to serve as a $\mathrm{SH}$. The helicopters of any swarm need to select a SH. The selection of the $\mathrm{SH}$ depends on different factors, such as communication equipment, velocity, and location within the swarm. Helicopter with satellite link has the highest equipment priority. Helicopter located in the center of the swarm also has the highest location priority.

In this paper for the purpose of $\mathrm{SH}$ election process, we make the following assumptions. The notation $P_{i}$ is the $\mathrm{SH}$ election priority factor (EPF). Each helicopter node has an ID number which is unique and known to the whole network. Each helicopter is equipped with a GPS used to periodically collect location, velocity, and direction. The maximum speed for each swarm member is $v_{\max }$. If the number of swarm members is too small, number of helicopter nodes that are able to act as a SH will increase and the network will become more isolated. If swarm members are too high the burden on the SH will increase. Consequently, the intraswarm communication cost increases, because the $\mathrm{SH}$ is responsible for communication between both its nodes and the neighbor SHs. Therefore, the swarm size needs certain constraints. In this paper, we define $M_{\max }$ to represent the maximum number of hops between the swarm members and the $\mathrm{SH}$. We also define $N_{\max }$ as the maximum number of nodes in each swarm. Furthermore, if the movement direction of the $\mathrm{SH}$ is opposite or different to most of the nodes within the swarm, the node quickly leaves the range of swarm and joins other swarms, which increases the swarm maintenance burden. Therefore, movement direction of the nodes needs to be taken into account when selecting SH. In actual scenarios, it is very difficult to find two nodes moving exactly with the same orientation. To solve this problem, we used a threshold $(\theta)$ to determine whether the directions are approximately the same or not. Two helicopters will be considered as moving in the same direction if the angle between them does not exceed the maximum thresholds. In this paper, helicopters are moving to the same direction and the maximum threshold of difference between two helicopters is 15 degrees. Helicopters 
moving in the opposite direction combine another swarm. SHs moving in different directions are able to connect as long as they were in the communication range. When they are outside the ad hoc coverage, they can communicate using satellite communication or through other SH. In order to avoid frequent changes in swarm structure, the speed of the nodes also is one of the important considerations. A $\mathrm{SH}$ should be the node relatively near to the average rate of other nodes velocity. Based on the above assumptions, a node is elected as a $\mathrm{SH}$ according to its EPF value; the following expresses the mathematical calculation of the EPF $\left(P_{i}\right)$.

Assume that $I$ represents the intended helicopter node, $J$ is another node in the same swarm, and each $I$ node has $N-1$ neighbors, $J_{1}, J_{2}, J_{3}, \ldots, J_{(N-1)}$, with coordinates $\left(x_{J_{1}}, y_{J_{1}}\right),\left(x_{J_{2}}, y_{J_{2}}\right), \ldots,\left(x_{J_{N-1}}, y_{J_{N-1}}\right)$. $\Phi_{I}$ is the group of nodes within the same swarm which are neighbors of node $I$. Intended helicopter coordinate is $x_{I}, y_{I}$. Helicopters velocities at instance election time are $v_{1}, v_{2}, \ldots, v_{N}$. Helicopters are classified according to their communication equipment into $S_{1}$ and $S_{2}$ as mentioned above.

Firstly, according to the equipment of the helicopter, the equipment priority value $P_{E}$ is as follows:

$$
P_{E}= \begin{cases}0 & \text { if type } S_{1} \\ 1 & \text { if type } S_{2}\end{cases}
$$

Helicopter with $P_{E}=0$ has highest equipment priority.

Secondly, to determine the helicopter which has the best location within the swarm, we used the summation of distances for each helicopter from all others. Distance between $I$ and $J$ nodes is obtained by

$$
D_{I, J}=\sqrt{\left(x_{I}-x_{J}\right)^{2}+\left(y_{I}-y_{J}\right)^{2}+\left(z_{I}-z_{j}\right)^{2}} .
$$

Each helicopter in the swarm has $(N-1)$ distances toward the others, which form the $\mathbf{D}_{I}$ distance set:

$$
\mathbf{D}_{I}=\left[\begin{array}{lllll}
D_{I 1} & D_{I 2} & D_{I 3} & \cdots & D_{I(N-1)}
\end{array}\right] .
$$

Summation of these distances for each node is obtained by

$$
S_{I}=\sum_{J=1}^{N-1} D_{I J}
$$

Obtaining $S_{I} \forall J \in \emptyset_{I}$ yields

$$
\mathbf{S}_{i}=\left[\begin{array}{lllll}
S_{1} & S_{2} & S_{3} & \cdots & S_{N}
\end{array}\right]
$$

where $S_{i}$ is distance values of all nodes in the swarm. The minimum value of the set $S_{i}$ is the best location. Helicopter with less value is the nearest one to all others and will take a high location priority in $\mathrm{SH}$ election. This means that the selected helicopter is not usually located in the centre of swarm, but it is the closest one to all swarm members. As the election process depends on many factors such as ID, equipment, and velocity priorities, we define another notation which is the location priority deviation factor $\left(P_{L}\right)$, where $P_{L}$ is associated with other priority factors to calculate the $\operatorname{EPF}\left(P_{i}\right)$ of a helicopter. It is a value used to represent the location priority deviation of a helicopter from that of best location priority. The following equations are used to calculate $\left(P_{L}\right)$ :

$$
\begin{aligned}
& S_{\mu}=\min \left(\mathbf{S}_{i}\right), \\
& P_{L}=S_{I}-S_{\mu} \quad \text { For node with minimum } \mathbf{S}_{I}, P_{L}=0 .
\end{aligned}
$$

$P_{L}$ of all nodes can be represented by

$$
\mathbf{P}_{\mathbf{L}_{\mathbf{I}}}=\left[P_{L_{1}}, P_{L_{2}}, P_{L_{3}}, \ldots, P_{L_{N}}\right] \text {. }
$$

Simply, it can be normalized by scaling between 0 and 1 using

$$
\operatorname{Normlized}\left(P_{L}\right)=\frac{P_{L}-\min \left(P_{L_{I}}\right)}{\max \left(\mathbf{P}_{\mathbf{L}_{\mathbf{I}}}\right)-\min \left(\mathbf{P}_{\mathbf{L}_{\mathbf{I}}}\right)} .
$$

Thirdly, to calculate the helicopter velocity priority, first simply calculate the mean velocity $v_{\mu}$ :

$$
v_{\mu}=\frac{1}{N} \sum_{i=1}^{N} v_{I}
$$

The velocity priority factor $P_{v}$ for each helicopter $(I)$ can be calculated as follows:

$$
\begin{aligned}
P_{v} & =\frac{1}{N}\left(v-v_{\mu}\right)^{2}, \\
\mathbf{P}_{\mathbf{v}_{\mathbf{I}}} & =\left[\begin{array}{lllll}
P_{v_{1}} & P_{v_{2}} & P_{v_{3}} & \cdots & P_{v_{N}}
\end{array}\right] .
\end{aligned}
$$

The set $\mathbf{P}_{\mathbf{v}_{\mathrm{I}}}$ represents the velocity priority factor of all helicopters. Node with the lowest $P_{v_{I}}$ has the highest velocity priority. The normalized velocity priority factor for each node can be

$$
\operatorname{Normlized}\left(P_{v}\right)=\frac{P_{v}-\min \left(\mathbf{P}_{\mathbf{v}_{\mathbf{I}}}\right)}{\max \left(\mathbf{P}_{\mathbf{v}_{\mathbf{I}}}\right)-\min \left(\mathbf{P}_{\mathbf{v}_{\mathbf{I}}}\right)} .
$$

Finally, the $\operatorname{EPF}\left(P_{i}\right)$ is a function of the normalized version of $P_{E}, P_{L}, P_{v}$ which are calculated in (2), (9), and (12), respectively. Every node calculates these values and then calculates the $\operatorname{EPF}\left(P_{i}\right)$ according to the following:

$$
P_{i}=a P_{E}+b P_{L}+c P_{v}
$$

where $a, b$, and $c$ are weighting values that satisfy $a+b+c=1$. The EPFs of all nodes are expressed by $\mathbf{P}_{\mathbf{i}_{\mathbf{I}}}$ :

$$
\begin{aligned}
\mathbf{P}_{\mathbf{i}_{\mathbf{I}}} & =\left[\begin{array}{lllll}
P_{i_{1}} & P_{i_{2}} & P_{i_{3}} & \cdots & P_{i_{N}}
\end{array}\right] \\
\operatorname{Normlized}\left(\mathbf{P}_{i}\right) & =\frac{\mathbf{P}_{i}-\min \left(\mathbf{P}_{\mathbf{i}_{\mathbf{I}}}\right)}{\max \left(\mathbf{P}_{\mathbf{i}_{\mathbf{I}}}\right)-\min \left(\mathbf{P}_{\mathbf{i}_{\mathbf{I}}}\right)} .
\end{aligned}
$$

(1) Each helicopter periodically sends a HELLO message (msg, hop_cnt) at the same time it forwards the HELLO messages of the other. Upon doing $k$ hops the node will be 
capable of processing other nodes' information, such as the basic specification and condition of the nodes, which include the ID number, the swarm election priority $\left(P_{i}\right)$, location information, velocity $(v)$, and hop count $(m)$. Every node upon receiving the HELLO message from others stores the information of the other nodes and subsequently uses this information to determine the hop count $(m)$ of each node and saves it for further usage. During the information collection process, the receiving node always determines the hop count of the other node, stores the original $m$, adds a number 1 to the count, and then forwards the information to the next node. Through this process, the next node will be able to determine the $m$ hop of that node; also it ensures that the number of hops does not exceed $M_{\max }$ and the receiving node avoids the looping. After a number of transmissions, the nodes collect their neighbors' information, including $m$ hops.

(2) After the collection of the information, each node (I) calculates their value $P_{i}(I)$ according to (13) and (15) and compares the result with $P_{i}(J)$ for all other nodes in the group $\Phi_{I}$, if

$$
P_{i}(I)=\min \left(P_{i_{I}}\right)
$$

The node with a lower $P_{i}$ value has the highest priority. Thus it will upgrade itself as a SH. If two or more nodes share the least value of $P_{i}$, hence

$$
\begin{aligned}
& P_{i}(I)=P_{i}(J)=\min \left(P_{i_{I}}\right) \\
& P_{E}(I)<P_{E}(J) .
\end{aligned}
$$

Node with least $P_{E}$ value is selected as a $\mathrm{SH}$, if

$$
\begin{aligned}
& P_{E}(I)=P_{E}(J) \\
& \mathrm{ID}(I)<\mathrm{ID}(J) .
\end{aligned}
$$

Node with the least ID should be elected as the SH.

(3) The helicopter, after upgrading itself as a $\mathrm{SH}$, changes its state to $S \_$head and broadcasts a message, HEAD (Swarm_ID, head_msg, route, hop_cnt), propagating swarm information, where Swarm_ID is a swarm label, head_msg is the $\mathrm{SH}$ node information, route is the route information, and hop_cnt is the number of hops. In the initial transmission, the value of route is set to null and hop_cnt is set to zero. Other nodes within the swarm, upon receiving the HEAD message, store all values and judge the value of hop_cnt. If hop_cnt is less than $M_{\max }$, then they will add one to this value and then forward the information to the next hop. If hop_cnt is equal to $M_{\max }$, then there is no need for further treatments. When other nodes receive the same Swarm_ID, they investigate the $m$ values and select the lowest one and then use this value to determine the value of their hop_cnt return. When they receive a message from a different $S w a r m_{-} I D$, they analyze head_msg of the SHs and the information of the high-priority node is retained.

(4) Nodes send apply message APPLY ((Swarm_ID), msg, route, hop_cnt) to the $\mathrm{SH}$ which has been selected according to steps (2) and (3). Other nodes on the route interrupt this message and update the route information by adding +1 to the hop_cnt and then forward the new route information to the $\mathrm{SH}$. This way $\mathrm{SH}$ can determine the hop count of its member.

(5) $\mathrm{SH}$ will add the node to its member list just after verifying that the number of nodes is less than $N_{\max }$. In this case, $\mathrm{SH}$ sends back an $A C C P$ message to the intended node but otherwise sends back a JOIN_GUEST message.

(6) Intended node after receiving the ACCP message broadcasts MEMR (Swarm_ID, msg, route, dis_cnt, hop_cnt) message and then is not needed to be involved in other election processes. It will send dis_cnt to other SHs.

(7) Repeat the process until all nodes become $\mathrm{SH}$ or swarm members.

(8) Border swarm members search for the adjacent node of the other swarm using the hop_cnt and Swarm_ID information making a list of all neighbor nodes.

(9) Each node broadcasts its neighbor table information periodically using HELLO packets. Therefore, by examining the neighbor table from its neighbors, a node is able to gather complete information about the network topology that is at most two hops away from itself. This two-hop topology information is kept in a small database in each node.

(10) Send a BUND (msg, route, dis_cnt, hop_cnt) message hop_cnt message to its $\mathrm{SH}$ indicating that it became a boundary node.

The SH and the neighbor's SH can communicate directly if they are located in the same coverage, but in the actual situation of the physical structure of the network the $\mathrm{SH}$ and the neighbor $\mathrm{SH}$ are not necessarily within the same communication range. For that reason $\mathrm{SH}$ selects one of the boundary swarm members to act as a gateway between the neighbor SHs. Therefore, $\mathrm{SH}$ election process not only is the election of the $\mathrm{SH}$ but also includes the operation of determining boundary nodes, used for communication between adjacent swarms, as shown in Figure 2.

After finishing $\mathrm{SH}$ election, the movement of nodes could change swarm structure. Therefore, swarm structure continuously needs maintenance. SH periodically checks member list and neighbors swarm listing status. In case of adding a new member or one of the member lefts, swarm structure will not change; $\mathrm{SH}$ only updates the members list. Sometimes due to the movement, the total number of nodes in two or more neighbor's swarms may detract from $N_{\max }$. In this case, the two swarms merge to form a new swarm. This situation leads to competition on $\mathrm{SH}$ using the same $\mathrm{SH}$ election process. Loser $\mathrm{SH}$ sends messages to inform its member about the new $\mathrm{SH}$ information. $\mathrm{SH}$ also updates the boundary nodes periodically. When a boundary node leaves the swarm, $\mathrm{SH}$ will select new border nodes. Nodes also periodically send message to ensure the presence of $\mathrm{SH}$ and update their information, particularly location and velocity. A new SH should be elected in case of loss or leaving of SH due to abnormal situation. Moreover, $\mathrm{SH}$ periodically calculates its $P_{i}$ and sends it to all members; if one or more members find that their $P_{i}$ value became less than that of the $\mathrm{SH}$, they will notify the $\mathrm{SH}$. SH immediately notifies its members about the new $\mathrm{SH}$. Other members respond accordingly by updating their SH information. The objective is to maintain the stability of the swarm structure, avoid exchanging many messages, 
and reduce performing many calculations. Therefore, the structure of the swarm will not be changed unless for the above mentioned situation or in case of deterioration in the SH performance.

\section{Information Routing}

Due to node mobility, a major challenge is how to route data packets over such network that changes its structure dynamically, especially when the source and the destination helicopters are out of MANET transmission range. Most of the MANET routing algorithms are not ideal for AVANETs, because of the helicopter specific issues such as rapid changes in the link quality and very high node mobility. Maintaining a routing table, as in proactive methods, also is not an optimal solution. However, repetitive path finding before each packet delivery, as in reactive routing, can also be exhaustive. Therefore, specific routing solutions are needed. A routing strategy only based on the location information of the nodes can satisfy the requirements of helicopter networks. Although some VANET routing protocols can work in these types of networks, majority of these protocols mainly rely on road side units (RSU) [29], road intersections [30], and other assumptions which may not be available in helicopters networks [31]. In this paper, we propose a simple routing algorithm which fits the special requirements of helicopter networks.

5.1. Route Finding. As a rule, helicopters located in the same swarm and satisfying (1) can directly use ad hoc networks to communicate with each other. In order to establish a communication between two nodes beyond the ad hoc coverage $(L)$, source node issues a communication request to $\mathrm{SH}$. SH after receipt of request verifies whether the node is one of the swarm members. After verification SH finds from its storage the location and other information of the destination node and then starts the process of best route selection, which is subject to destination and source locations. SH is not only responsible of the route selection process; in many situations $\mathrm{SH}$ can participate in information forwarding process.

In case of the fact that the intended node is one of the swarm members, $\mathrm{SH}$ searches the best route according to the following. The presence of multiple nodes between source and destination helicopters may lead to many paths $(P)$. Each path has its own amount of hops $M_{P} . d_{m}$ is the distance of a specific hop $m$, and $d_{m}$ satisfies (1), where $M_{P} \leq M_{\max }$. Therefore, the general formula for short path selection is

$$
P_{s}=\min \left[\sum_{m=1}^{M_{1}} d_{m}, \sum_{m=1}^{M_{2}} d_{m}, \sum_{m=1}^{M_{3}} d_{m}, \ldots, \sum_{m=1}^{M_{P}} d_{m}\right] .
$$

For simplicity, assume that we have five nodes $\mathrm{SH}, I$, $K, J$, and $D$, as shown in Figure 3. Assume that their locations coordinates are $\left(x_{\mathrm{SH}}, y_{\mathrm{SH}}, z_{\mathrm{SH}}\right),\left(x_{I}, y_{I}, z_{I}\right),\left(x_{k}, y_{K}, z_{K}\right)$, $\left(x_{J}, y_{J}, z_{J}\right)$, and $\left(x_{d}, y_{d}, z_{d}\right)$, respectively, where $I$ is the source node, $D$ is the destination node, and $J, k$ are nodes in the same coverage $(L)$ of SH. $J$ and $K$ act as a next hop. Distance $(K, \mathrm{SH}),(I, \mathrm{SH}),(K, I)$, and $(K, D)$ satisfy $(1)$. SH finds

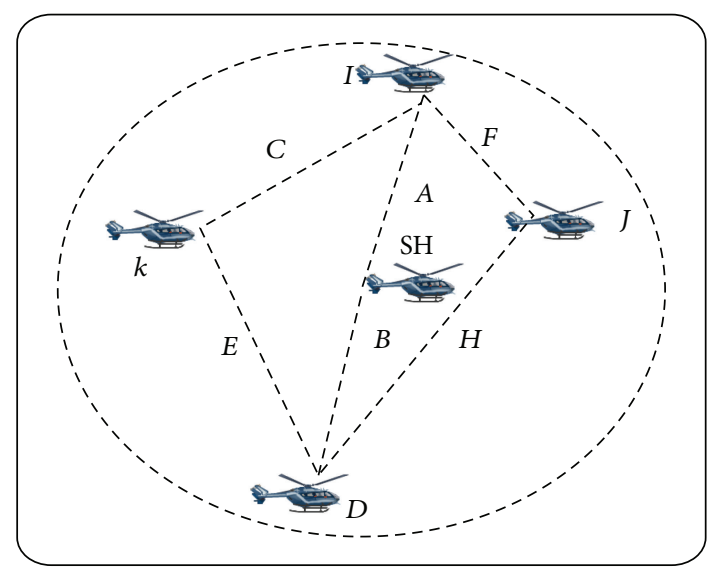

FIgURE 3: Information routing inside swarm.

the information of these nodes and starts calculating the distance according to the following:

$$
\begin{aligned}
& A=\sqrt{\left(x_{I}-x_{\mathrm{SH}}\right)^{2}+\left(y_{I}-y_{\mathrm{SH}}\right)^{2}+\left(z_{I}+z_{\mathrm{SH}}\right)^{2}}, \\
& B=\sqrt{\left(x_{\mathrm{SH}}-x_{D}\right)^{2}+\left(y_{\mathrm{SH}}-y_{D}\right)^{2}+\left(z_{\mathrm{SH}}-z_{D}\right)^{2}}, \\
& C=\sqrt{\left(x_{I}-x_{K}\right)^{2}+\left(y_{I}-y_{K}\right)^{2}+\left(z_{I}-z_{K}\right)^{2}}, \\
& E=\sqrt{\left(x_{K}-x_{D}\right)^{2}+\left(y_{K}-y_{D}\right)^{2}+\left(z_{K}-z_{D}\right)^{2}}, \\
& F=\sqrt{\left(x_{I}-x_{J}\right)^{2}+\left(y_{I}-y_{J}\right)^{2}+\left(z_{I}-z_{J}\right)^{2}}, \\
& H=\sqrt{\left(x_{J}-x_{D}\right)^{2}+\left(y_{J}-y_{D}\right)^{2}+\left(z_{J}-z_{D}\right)^{2}},
\end{aligned}
$$

where $A, B, C, E, F$, and $H$ are the distances between nodes as shown in Figure 3 . Shorter path $\left(P_{s}\right)$ to the destination node is

$$
P_{s}=\min \{A+B, C+E, F+H\} .
$$

In case of the fact that the $\mathrm{SH}$ does not find the destination information on its storage, it requests the closest SHs for the destination information and waits for their responses. If the waiting time exceeds a threshold $\left(t_{r}\right)$ and the route response has not yet been received, then $R R E Q$ is resent. If retransmission exceeds the maximum retransmission limit $\left(r_{\max }\right)$, the route search process is terminated. To reduce network congestion, not all neighbor $\mathrm{SHs}$ receiving RREQ will respond. Only those located on the route toward the destination and having the ability to serve can participate in the routing process.

Therefore, neighbor SHs after having received RREQ message firstly determine if they are located on the route of the $\mathrm{SH}$ request; if not, the request is discarded. Otherwise the procedure is as follows:

(1) A neighbor SH checks the request; if it is the first time received, then it continues the procedure; if not then the request is discarded. 
(2) It checks whether the destination is in the same swarm. If not, then it applies step (4).

(3) It forwards route request to the intended destination and waits for the routing response RREP; it also goes to (5).

(4) SH adds its own msg to (REEQ msg) message and then forwards the updated message to the SHs of the next hope neighbours and waits for the route response.

(5) If the waiting time exceeds $t_{r}$ and it does not receive the route response, then it retransmits request and adds one to request number; otherwise it goes to (7).

(6) If the number of retransmissions exceeds the limit $r_{\text {max }}$, then it ends route request process. Alternatively, in accordance to intended destination situation it directly applies step (3) or (4).

(7) If the $\mathrm{SH}$ received more than one route response, then it chooses the node with fewest number of hops and minimum distance to destination and adds its msg to (REEP msg) message. Then, it forwards the message to the previous hop $\mathrm{SH}$. If the routing request failed, probably because the destination swarm is far away from the source and the routing and communication request cannot be established through ad hoc or through neighbours' $\mathrm{SH}$, the $\mathrm{SH}$ can establish a connection via satellite or other direct links.

(8) In some rare situations and according to SH election process, $\mathrm{SH}$ may not have a satellite equipment to communicate with the far away swarm's heads; in this case the $\mathrm{SH}$ finds one of its swarm members which are equipped with satellite, to forward request to neighbors $\mathrm{SH}$.

(9) If still not able to establish connection and routing through satellite of the members, it forwards request to neighbors $\mathrm{SH}$ and it sends notification WAIT indicating that the communication needs to be completed through satellite link of neighbors $\mathrm{SH}$.

Nodes usually prefer the use of ad hoc connections, but if the destination is out of the coverage, a node needs to communicate with operation center or public networks. In these cases, the source $\mathrm{SH}$ firstly searches its members to find a member that is equipped with an appropriate communication link to the intended network. If all swarm members are not equipped with suitable equipment, then $\mathrm{SH}$ forwards the request to the neighbor $\mathrm{SH}$ that is equipped with the appropriate communication equipment. Only helicopters type $S_{1}$ can communicate with the outside networks. Upon receiving a communication request to outside networks, neighbor $\mathrm{SH}$ will proceed as follows:

(1) It check the request. If it is the first time received, it continues the procedure; if not, then it is discarded.

(2) It checks whether its equipment is of type $S_{2}$; then it will skip to step (4).

(3) It checks whether this swarm has a free available $S_{1}$ and then forwards the routing request, waits for response, and then skips to step (5).
(4) It forwards the routing request to the neighboring $\mathrm{SH}$ and waits for a response.

(5) If the waiting time exceeds $t_{r}$ and it does not receive the route response, then it will retransmit the request and add 1 to the request number; otherwise it will skip to step (7).

(6) If the number of retransmissions exceeds the limit $r_{\max }$, then it will end the route request process.

(7) If the $\mathrm{SH}$ receives more than one route response, then it chooses the helicopter with fewest numbers of routing hops and the minimum distance and cost to the destination. The route through this node will be marked as the primary route. At the same time, it stores other routes as a backup (Store Route as Backup $(S R B))$ to be used in the future if needed. To ovoid looping multiple links to the same destination cannot be used at the same time. Any redundant link that is not as preferred is blocked until the primary link goes down.

(8) It adds its own msg to (REEP msg) message and forwards the message to the previous hop $\mathrm{SH}$ in the source direction. If the routing process fails to find a helicopter type $S_{1}$, it will send a REER notification to the source helicopter indicating a routing failure.

5.2. Route Maintenance (RM). According to nodes' movement the established route may be lost. This time, the communication will temporarily be disconnected. At the same time, the node stores the destination routing information for a while and tries to resend the request. If it is successful in sending a new route request to the intended destination, that means that the route has been recovered; if not, then it will search its backup routing information and try to use one of the best backup routes. If it fails to send the message it will start a new route finding procedure. The route maintenance also can be accomplished by using the knowledge of the nodes about their neighbor status. A forwarding node can aid in the route maintenance process by repairing a broken route using its 2-hop-topology information and modifies the source route header accordingly. The destination node sends a gratuitous route reply to inform source of the new working route.

\section{Simulation and Results}

6.1. Simulation Configurations and Settings. To evaluate the proposed work, we conducted two different types of simulations. Firstly, we evaluate the SH election approach using a Matlab program. Secondly, to evaluate the performance of the proposed routing protocol, we used the well-known simulation program OPNET $[15,32,33]$, which is a discrete event network simulator that includes a rich set of detailed models for ad hoc networks. As this work does not consider the physical and data link layer the simulation program is built on the specification of IEEE 802.11p standard. The main parameters of the simulations are summarized in Table 1.

In the first simulation, we implemented a simulation program to demonstrate the velocity, equipment, location, 
TABLE 1: Simulation settings and configurations.

\begin{tabular}{lc}
\hline Parameters & Values \\
\hline Packet size & 1024 \\
Channel sensing & 0.1 \\
Reception power thresholds & $0.5 \mathrm{~W}$ \\
Traffic type & Poison \\
Simulation time & $1200 \mathrm{~s}$ \\
Coverage area & $5000 \times 5000(\mathrm{~m})$ \\
$L$ & $1000 \mathrm{~m}$ \\
$M_{\max }$ & $5 \mathrm{hop}$ \\
$N$ & 15 node \\
Simulation period & 20 minutes \\
\hline
\end{tabular}

and movement direction of the helicopters. The purpose of the program is to show the $\mathrm{SH}$ election process according to the proposed election approach. In this simulation, we assume a number of helicopters in one swarm moving toward the same direction with different velocities, location, and equipment values. We assume that the velocity and location of all helicopters are randomly distributed and the starting point is randomly selected. In addition, we supposed that the number of helicopters of types $S_{1}$ and $S_{2}$ is $20 \%$ and $80 \%$, respectively. The simulation is executed several times using different number of helicopter nodes to calculate the different value of priority factors. The priority factors versus the ID numbers of helicopters are plotted in different situations.

In the second simulation, we used OPNET to compare the performance of the proposed routing algorithm with both CBRP [22] and Dynamic Source Routing (DSR) [25] protocols. As the proposed routing protocol has both the on-demand and clustering characteristics, we select these protocols for compression because of the on-demand properties of DSR and the clustering characteristics of CBRP. These protocols also demonstrated acceptable performance in vehicular networks. All protocols are operated at $12 \mathrm{Mbps}$ data rate. The power transmit level of $1 \mathrm{mw}$ was used for all scenarios. The nodes are moving in the simulation area according to two scenarios. In the first one, helicopters are moving according to a random waypoint model. Each node chooses a random location destination and moves toward it at a speed randomly distributed between 0 and $500 \mathrm{~km} / \mathrm{h}$. In the second scenario a group of helicopters are moving in regular swarm with a constant speed $200 \mathrm{Km} / \mathrm{h}$. The regular swarm is constructed by following three rules, which are move in the same direction as your neighbors, remain close to your neighbors, and avoid collisions with your neighbors. All helicopter nodes in the network are configured to run the proposed routing algorithm during the first round. Then they used DSR and CBRP during the second and third rounds, respectively. In case of CBRP, we treated the helicopter swarm as a cluster and helicopter with the least ID number as a cluster head. Since the elected SH plays a major role during the routing process, we used it during the evaluation of the proposed routing algorithm. The performance of the proposed algorithm is evaluated against DSR and CBRP in terms of swarm structure reconstructions, Packet Delivery

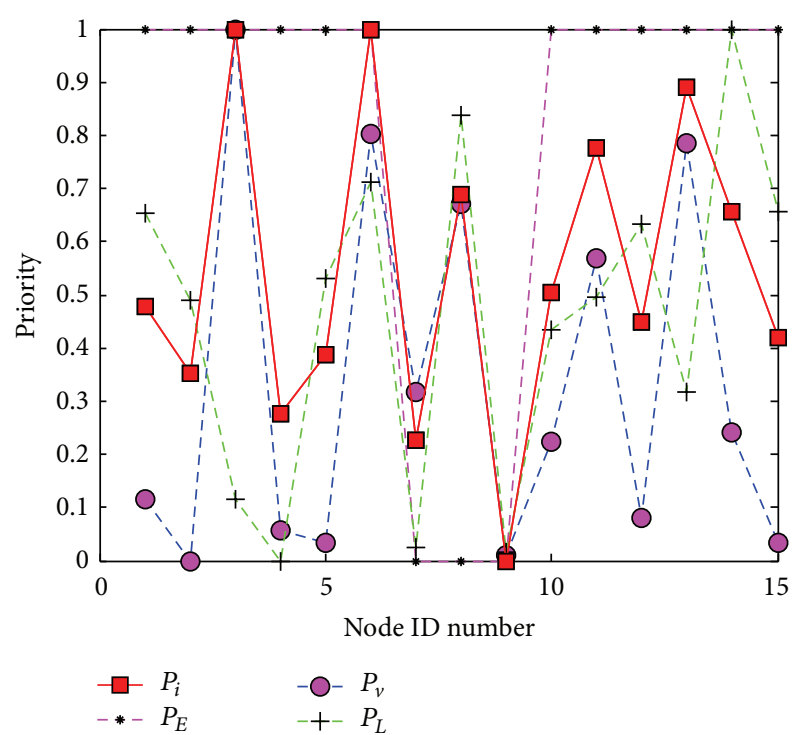

FIGURE 4: SH election priority. Node with the least $\operatorname{EPF}\left(P_{i}\right)$ is elected as a $\mathrm{SH}(\mathrm{ID}=9)$.

Ratio (PDR), average end-to-end delay (AD), and routing cost.

6.2. Results and Analysis. Simulation results are shown in Figures 4-9. The graphs show the election procedure result and the comparison between the three mentioned protocols using different parameter values. Figure 4 shows the priority factors (location, velocity priority equipment, and $\mathrm{EPF}$ ) of helicopter nodes. From Figure 4, node ID number nine is elected as a SH according to EPF. Obviously, in case of using only velocity to elect the $\mathrm{SH}$, as many other routing protocols do [34], node ID number two will be elected as SH though it is type $S_{2}$. Likewise, in case of using the location to elect the $\mathrm{SH}$, there are three nodes $(4,7$, and 9) with the same location priority; therefore, it will be difficult to elect $\mathrm{SH}$ among them. In order to achieve a better utilization of all nodes and optimum selection the use of EPF will solve the problem by using a weighted mechanism. This problem has not been addressed by other existing protocols that used location or velocity to elect the $\mathrm{SH}$.

Furthermore, to demonstrate that the proper election of the $\mathrm{SH}$ has a great effect on the stability of the network, we run the simulation using the proposed routing algorithm with $\mathrm{SH}$ elected using one priority factor (velocity, location, equipment, or EPF), and then we monitor the swarm structure every $100 \mathrm{~s}$. The accumulated number of the swarm reconstruction is plotted in Figure 5. It is realized that, using $\mathrm{SH}$ which is elected according to $P_{E}$, the network structure is better than when using velocity or location to elect $\mathrm{SH}$. However, sometimes the variation in the velocity of the wellequipped nodes affects its performance and consequently affects the network structure. The effect of $P_{L}$ and $P_{v}$ seems to be convergent because the velocity affects the location of the nodes. When EPF is used, the swarm structure is more stable, because the selection process considers all factors. 


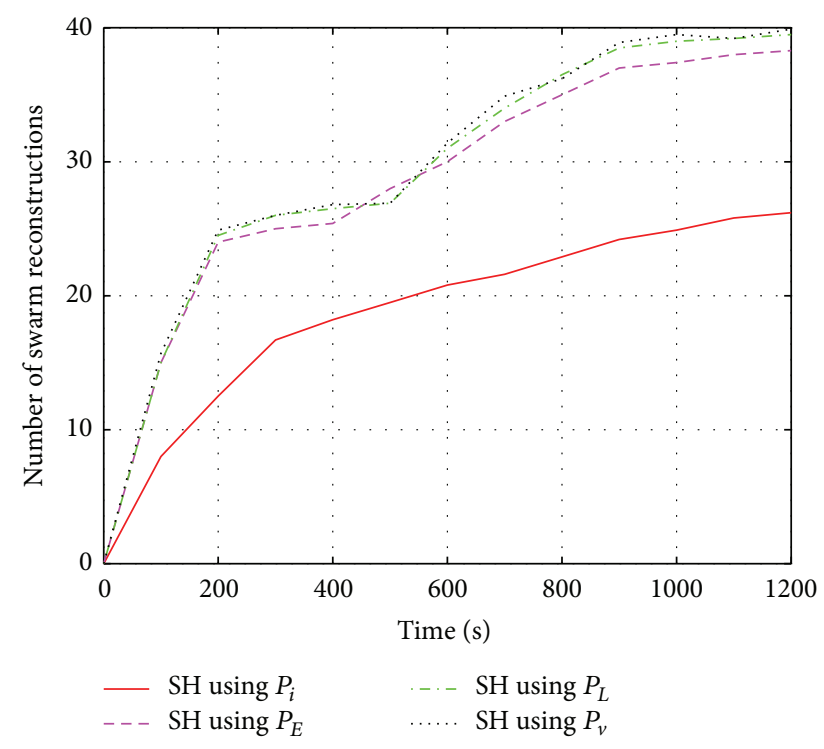

FIGURE 5: The effect of SH election process in swarm stability.

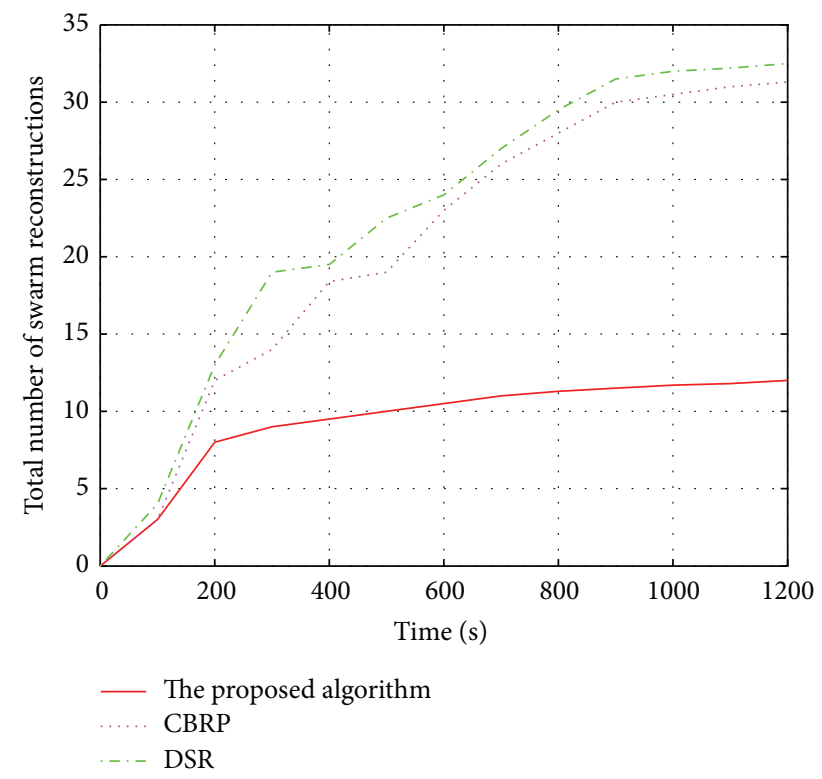

FIGURE 6: Swarm structure stability over time for the proposed, CBRP, and DSR protocols.

We also investigate the swarm reconstruction using the CBRP, DSR, and the proposed algorithm. As shown in Figure 6, the swarm structure stability of both CBRP and DSR is poor. This is because CBRP algorithm uses only the IDs of the nodes as a basis for SH election. The smallest ID is elected as a $\mathrm{SH}$. A swarm structure may change rapidly in case of the fact that the elected node moves with high velocity. On the other hand, using DSR, a separate periodic algorithm must be implemented, to support propagating the $\mathrm{SH}$ advertisements across the swarm. The response of the algorithm may affect the network structure. In contrast, the proposed election process takes into consideration the movement, location,

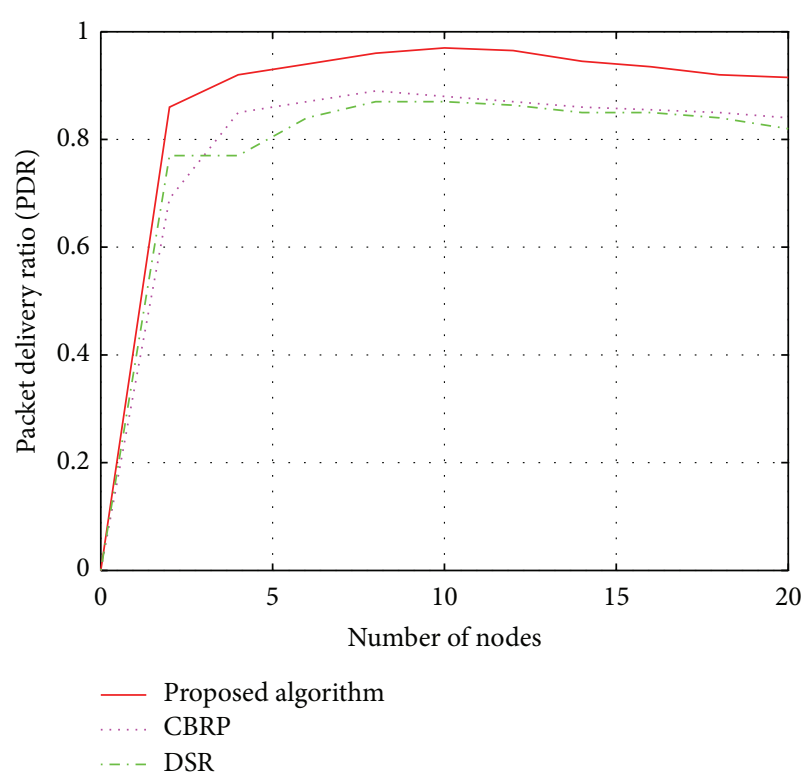

FIGURE 7: The PDR of the DSR, CBRP, and proposed algorithms.

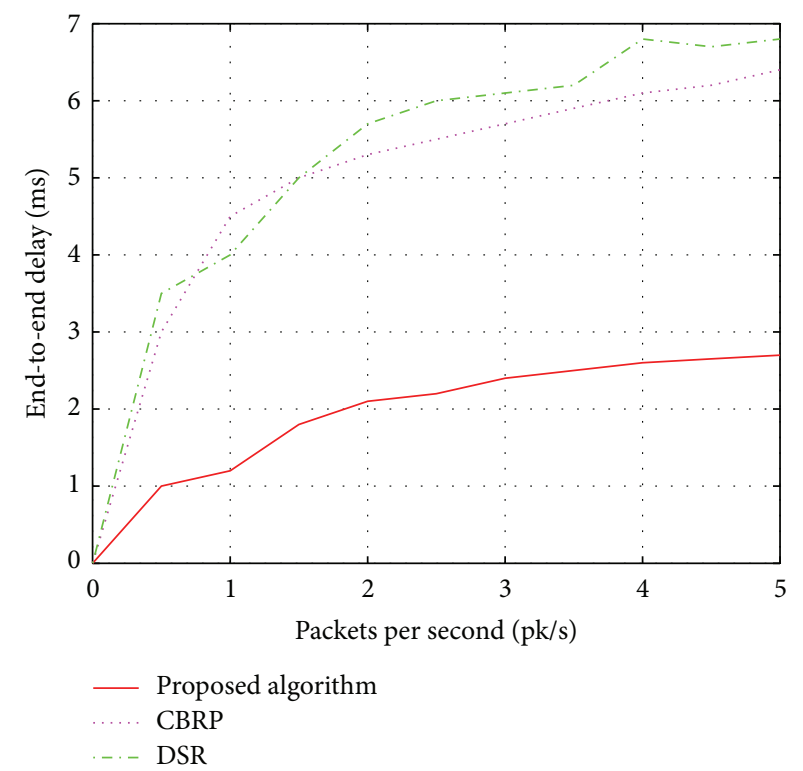

FIGURE 8: The proposed algorithm, CBRP, and DSR number of sent packets versus end-to-end delay for 15 helicopters.

and equipment of neighbouring helicopters, to reduce the probability of swarm change over time, so as to maintain higher stability.

It is observed from Figure 7 that the PDR of the proposed algorithm remains high when the number of nodes is increasing. The increase of the number of nodes does not affect the PDR because of the high efficiency and swarm structure stability. PDR of CBRP and DSR is less than that obtained by the proposed algorithm, because the source node and the intermediate nodes store the next hop information corresponding to each flow for data packet transmission. DSR and CBRP use a source routing in which a data packet carries 


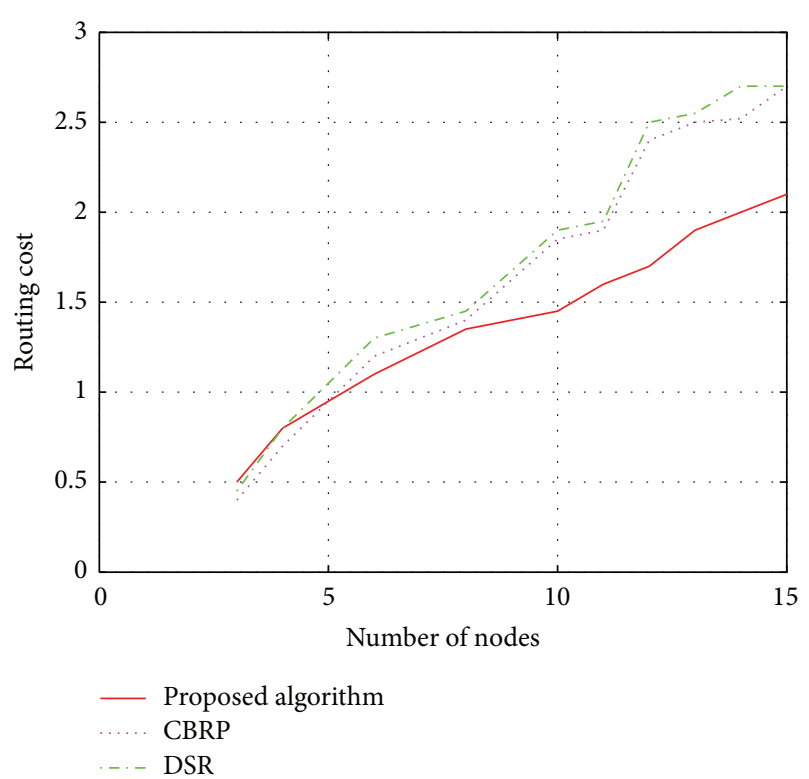

FIGURE 9: Proposed algorithm, CBRP, and DSR routing cost versus number of helicopters.

the complete path to be traversed. The PDR is too low in the beginning of the curve due to the random initiation of the simulation program.

Concerning Figure 8, we observed that the average endto-end delay of the proposed algorithm is the best among others. The proposed algorithm end-to-end delay is smaller than the others because the route search is done only once and remains stable until the swarm structure changes. This decreases the time needed for the whole process of packet delivery and reduces the number of routing messages. Other protocols have a longer delay because the route finding process takes more time as every intermediate node tries to extract information before forwarding the reply while the protocol tries to search for a new route. The proposed algorithm also outperforms others because of the built-in RM and SRB characteristics. While using other protocols, the routing mechanism searches for a new route at every request and when a route failure occurs. The packet delivery delay time is affected by both the route search algorithm and the packet delivery process itself.

According to Figure 9, it is observed that the routing cost of the proposed algorithm, CBRP and DSR, increases with respect to the increase of the number of nodes. This is because the increase in nodes quantity increases the amount of hops, thereby increasing the congestion in the routing process. The initiation of the route finding process of the proposed algorithm takes more time. However, this calculation is done once and remains steady until the swarm structure changes. Hopefully, the increase of swarm members will increase the opportunity of more backup route in the proposed algorithm that enhances the route maintenance process. Moreover, the number of nodes per swarm and the number of hops in the proposed algorithm are limited by $N_{\max }$ and $M_{\max }$, respectively. The amount of $N_{\max }$ and $M_{\max }$ is specified according to certain threshold of the routing cost.
The problem of other routing protocols is that the increase of intermediate nodes can lead to inconsistent routes when the source sequence number is very old and the intermediate nodes have a higher but not the latest destination sequence number, thereby having no existing or stale entries. CBRP and DSR have a higher routing cost, because they use a flooding approach to all nodes to determine the destination route, while in the proposed routing algorithm only SHs in the requested swarm that have the capability of finding the destination are able to participate in the routing process.

\section{Conclusions}

This paper studied the possibility of using MANET as a platform for data communication between helicopters working in certain mission and special scenarios. A simple model for the network structure is proposed and intensively explained. A simple routing protocol is also presented and comprehensively evaluated using simulation. Theoretical approach for selecting the $\mathrm{SH}$ is proposed and extensively discussed. The investigation results show that ad hoc network can be involved in providing communication between helicopters with high degree of reliability and minimum expense. Our comprehensive simulation results show that the proposed $\mathrm{SH}$ election approach can assist in obtaining best results of SH selection, so as to maintain high network stability. The proper selection has great impact in the structure stability of the swarm and consequently affects the whole performance of the network. Simulation results also show that the proposed routing algorithm can be used in these types of networks with high delivery rate and less amount of delay and routing overhead. Generally this work sets the path for many researches that are related to utilization of MANET in helicopter networks. Many researches related to the PHY, data link, routing, and application of AVANET are desired. Although the general aspects are considered in this paper, there is still a lot of work and further detailed research can be conducted in more depth. Future researches can focus on studying the capability of using bidirectional routing and enhancing the $\mathrm{SH}$ election process by adding more priority factors such as signal strength and the movement stability of nodes as well as considering security issues. Many researches and efforts can focus on improving the physical link performance in order to increase the coverage range of a single hop. Future work can also consider obtaining actual results by implementing a kind of real practical deployment.

\section{Conflict of Interests}

The authors declare that there is no conflict of interests regarding the publication of this paper.

\section{Acknowledgments}

This work is supported by National Natural Science Foundation of China (no. 610011024) and the National Key Technology R\&D Program under Grant 2012BAH15B00. 


\section{References}

[1] E. C. Eze, S. Zhang, and E. Liu, "Vehicular ad hoc networks (VANETs): current state, challenges, potentials and way forward," in Proceedings of the 20th International Conference on Automation and Computing (ICAC '14), pp. 176-181, Cranfield, UK, September 2014.

[2] C. Elliott and B. Heile, "Self-organizing, self-healing wireless networks," in Proceedings of the IEEE Aerospace Conference, pp. 149-156, Big Sky, Mont, USA, March 2000.

[3] C. Prabha, D. S. Kumar, and D. R. Khanna, "Wireless multihop ad-hoc networks: a review," IOSR Journal of Computer Engineering, vol. 16, no. 2, pp. 54-62, 2014.

[4] N. Gondaliya and D. Kathiriya, "An application of ad hoc networks in disaster area for search and rescue operation: a survery and challenges," International Journal of Advanced Research in Computer and Communication Engineering, vol. 3, no. 3, pp. 5711-5714, 2014.

[5] A. M. Abdelgader and W. Lenan, "The physical layer of the IEEE 802.11 p WAVE communication standard: the specifications and challenges," in Proceedings of the World Congress on Engineering and Computer Science (WCECS '14), San Francisco, Calif, USA, October 2014.

[6] J. Schlecht, K. Altenburg, B. M. Ahmed, and K. E. Nygard, "Decentralized search by unmanned air vehicles using local communication," in Proceedings of the International Conference on Artificial Intelligence (IC-AI '03), pp. 757-762, June 2003.

[7] R. Haagensen, K.-A. Sjøborg, A. Rossing, H. Ingilæ, L. Markengbakken, and P.-A. Steen, "Long-range rescue helicopter missions in the arctic," Prehospital and Disaster Medicine, vol. 19, no. 2, pp. 158-163, 2004.

[8] K. Jusoff, "Search and rescue (SAR) operations for the missing Bell 206 Long Ranger helicopter in Sarawak, Malaysia using near real-time airborne hyperspectral imaging system," Disaster Prevention and Management, vol. 17, no. 1, pp. 94-103, 2008.

[9] R. A. F. Master Aircrew Duncan Tripp RAF, Working with Search \& Rescue Helicopters, RAF SAR Force Version 1.0, vol. 1, 2011.

[10] I. Bekmezci, O. K. Sahingoz, and \$̧. Temel, "Flying ad-hoc networks (FANETs): a survey," Ad Hoc Networks, vol. 11, no. 3, pp. 1254-1270, 2013.

[11] Y. Chen, K. L. Moore, and Z. Song, "Diffusion boundary determination and zone control via mobile actuator-sensor networks (MAS-net): challenges and opportunities," in Intelligent Computing: Theory and Applications II, vol. 5421 of Proceedings of SPIE, pp. 102-113, Orlando, Fla, USA, April 2004.

[12] P. K. Branch, "Unmanned vehicles: mainstays of future airborne reconnaissance," in Airborne Reconnaissance XXIII, vol. 3751 of Proceedings of SPIE, pp. 36-40, Denver, Colo, USA, December 1999.

[13] B. Argrow, D. Lawrence, and E. Rasmussen, "UAV systems for sensor dispersal, telemetry, and visualization in hazardous environments," in Proceedings of the 43rd AIAA Aerospace Sciences Meeting and Exhibit, pp. 15097-15107, Reno, Nev, USA, January 2005.

[14] Y.-J. Eun and H. Bang, "Cooperative control of multiple UCAVs for suppression of enemy air defense," in Proceedings of the AIAA 3rd Unmanned Unlimited Technical Conference, Workshop and Exhibit, vol. 6529, Chicago, Ill, USA, September 2004.

[15] A. I. Alshbatat and L. Dong, "Performance analysis of mobile ad hoc unmanned aerial vehicle communication networks with directional antennas," International Journal of Aerospace Engineering, vol. 2010, Article ID 874586, 14 pages, 2010.
[16] P. Gaudiano, B. Shargel, E. Bonabeau, and B. T. Clough, "Swarm intelligence: a new $\mathrm{c} 2$ paradigm with an application to control swarms of uavs," DTIC Document, 2003.

[17] D. L. Gu, G. Pei, H. Ly, M. Gerla, B. Zhang, and X. Hong, "UAV aided intelligent routing for ad-hoc wireless network in single-area theater," in Proceedings of the IEEE Wireless Communications and Networking Conference (WCNC '00), pp. 1220-1225, Chicago, Ill, USA, September 2000.

[18] S. Levy, "How Google will use high-flying balloons to deliver internet to the hinterlands," Wired, 2013.

[19] F. Lardinois, Google X Announces Project Loon: Balloon-Powered Internet for Rural, Remote and Underserved Areas, vol. 14, TechCrunch/AOL, 2013.

[20] K. Schulze and J. Buescher, "A scalable, economic autonomous flight control and guidance package for UAVs," in Proceedings of the 2nd AIAA "Unmanned Unlimited" Systems, Technologies and Operations-Aerospace, pp. 15-18, San Diego, Calif, USA, September 2003.

[21] K. Xu, X. Hong, M. Gerla, H. Ly, and D. L. Gu, "Landmark routing in large wireless battlefield networks using UAVs," in Proceedings of the IEEE Communications for Network-Centric Operations: Creating the Information Force (MILCOM '01), pp. 230-234, IEEE, October 2001.

[22] M. Jian, J. Li, and Y. C. Tay, "Cluster based routing protocol (CBRP) functional specification," Internet Draft draft-ietfmanet-cbrp-spec-00.txt, 1998.

[23] W. Chujo, "Helicopter satellite communication system for disaster control operations," in Proceedings of the 6th AsiaPacific Seminar on Next Generation Mobile Communications, 2005.

[24] T. Samad, J. S. Bay, and D. Godbole, "Network-centric systems for military operations in urban terrain: the role of UAVs," Proceedings of the IEEE, vol. 95, no. 1, pp. 92-107, 2007.

[25] D. Johnson, Y. Hu, and D. Maltz, "The dynamic source routing protocol (DSR) for mobile ad hoc networks for IPv4," RFC 47282007, 2007.

[26] T. Taleb, E. Sakhaee, A. Jamalipour, K. Hashimoto, N. Kato, and Y. Nemoto, "A stable routing protocol to support ITS services in VANET networks," IEEE Transactions on Vehicular Technology, vol. 56, no. 6, pp. 3337-3347, 2007.

[27] O. Abedi, M. Fathy, and J. Taghiloo, "Enhancing AODV routing protocol using mobility parameters in VANET," in Proceedings of the IEEE/ACS International Conference on Computer Systems and Applications (AICCSA '08), pp. 229-235, Doha, Qatar, April 2008.

[28] B. Kate, J. Waterman, K. Dantu, and M. Welsh, "Simbeeotic: a simulator and testbed for micro-aerial vehicle swarm experiments," in Proceedings of the 11th ACM/IEEE Conference on Information Processing in Sensing Networks (IPSN '12), pp. 4960, Beijing, China, April 2012.

[29] K. Mershad, H. Artail, and M. Gerla, "ROAMER: roadside Units as message routers in VANETs," Ad Hoc Networks, vol. 10, no. 3, pp. 479-496, 2012.

[30] J. Nzouonta, N. Rajgure, G. Wang, and C. Borcea, "VANET routing on city roads using real-time vehicular traffic information," IEEE Transactions on Vehicular Technology, vol. 58, no. 7, pp. 3609-3626, 2009.

[31] K. C. Lee, U. Lee, and M. Gerla, "Survey of routing protocols in vehicular ad hoc networks," Advances in Vehicular Ad-Hoc Networks: Developments and Challenges, pp. 149-170, 2010.

[32] W. Wang and J. Zhang, OPNET Modeler and Network Simulation, Posts \& Telecom Press, Beijing, China, 2003. 
[33] G. F. Lucio, M. Paredes-Farrera, E. Jammeh, M. Fleury, and M. J. Reed, "OPNET modeler and NS-2: comparing the accuracy of network simulators for packet-level analysis using a network testbed," WSEAS Transactions on Computers, vol. 2, pp. 700-707, 2003.

[34] K.-T. Feng, C.-H. Hsu, and T.-E. Lu, "Velocity-assisted predictive mobility and location-aware routing protocols for mobile Ad Hoc networks," IEEE Transactions on Vehicular Technology, vol. 57, no. 1, pp. 448-464, 2008. 


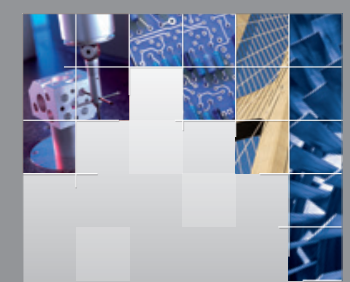

\section{Enfincering}
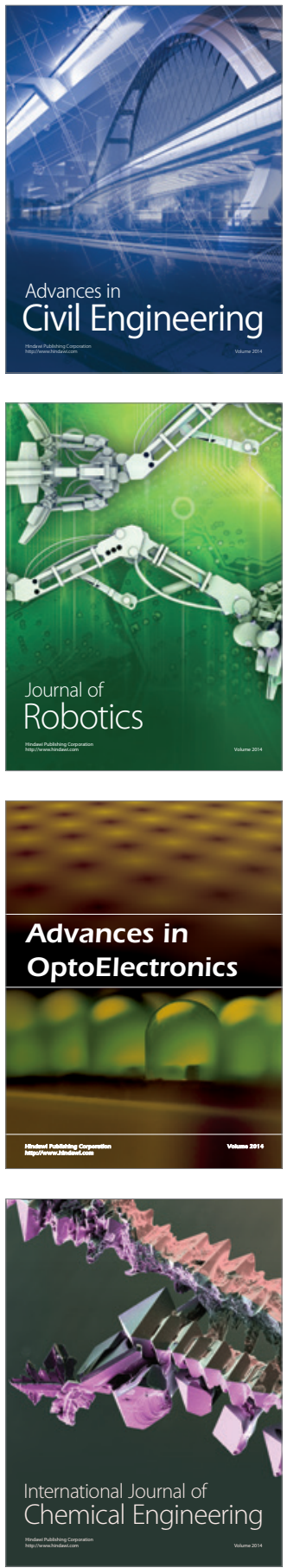

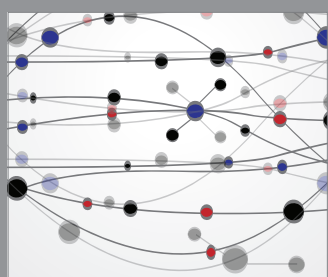

The Scientific World Journal

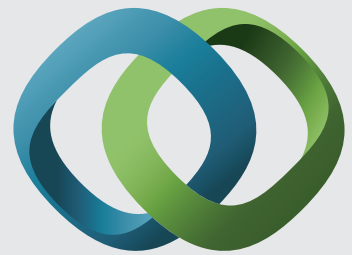

\section{Hindawi}

Submit your manuscripts at

http://www.hindawi.com
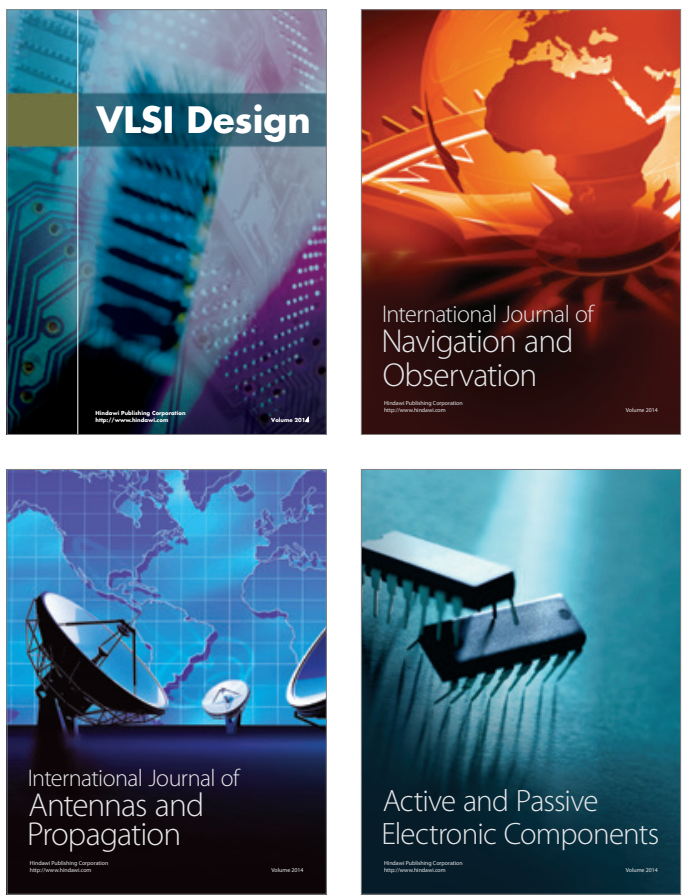
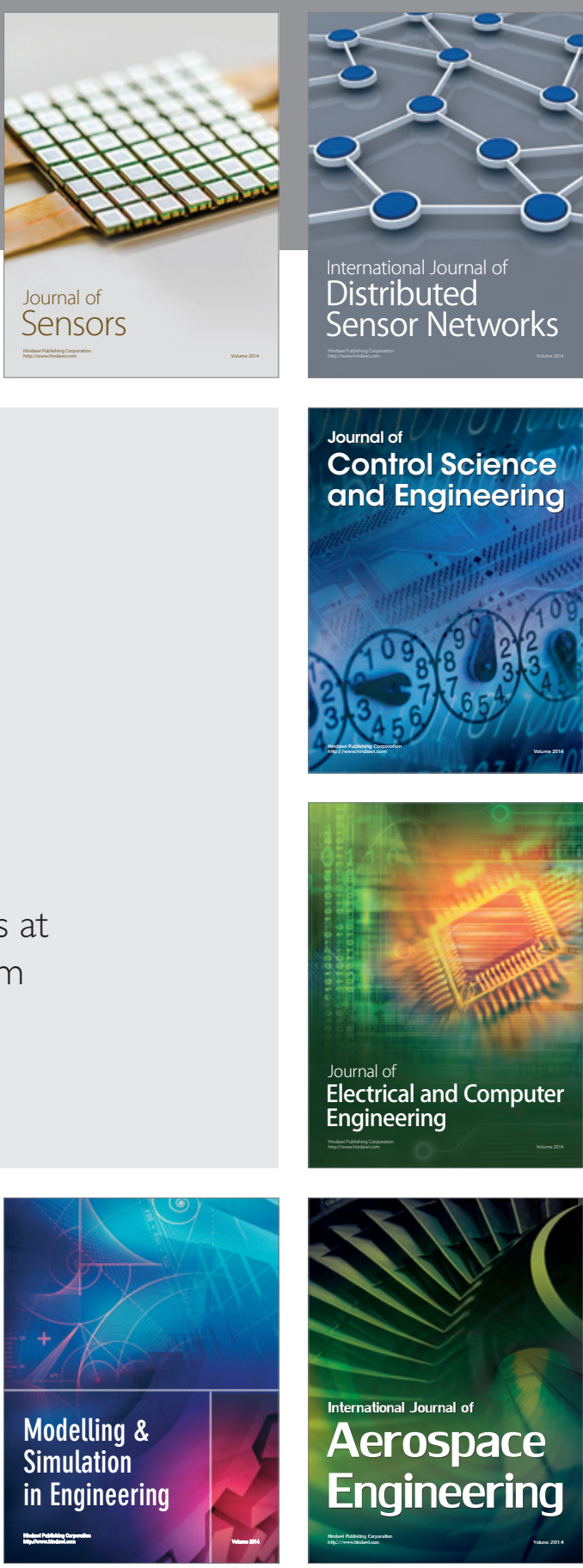

International Journal of

Distributed

Sensor Networks

Journal of

Control Science

and Engineering
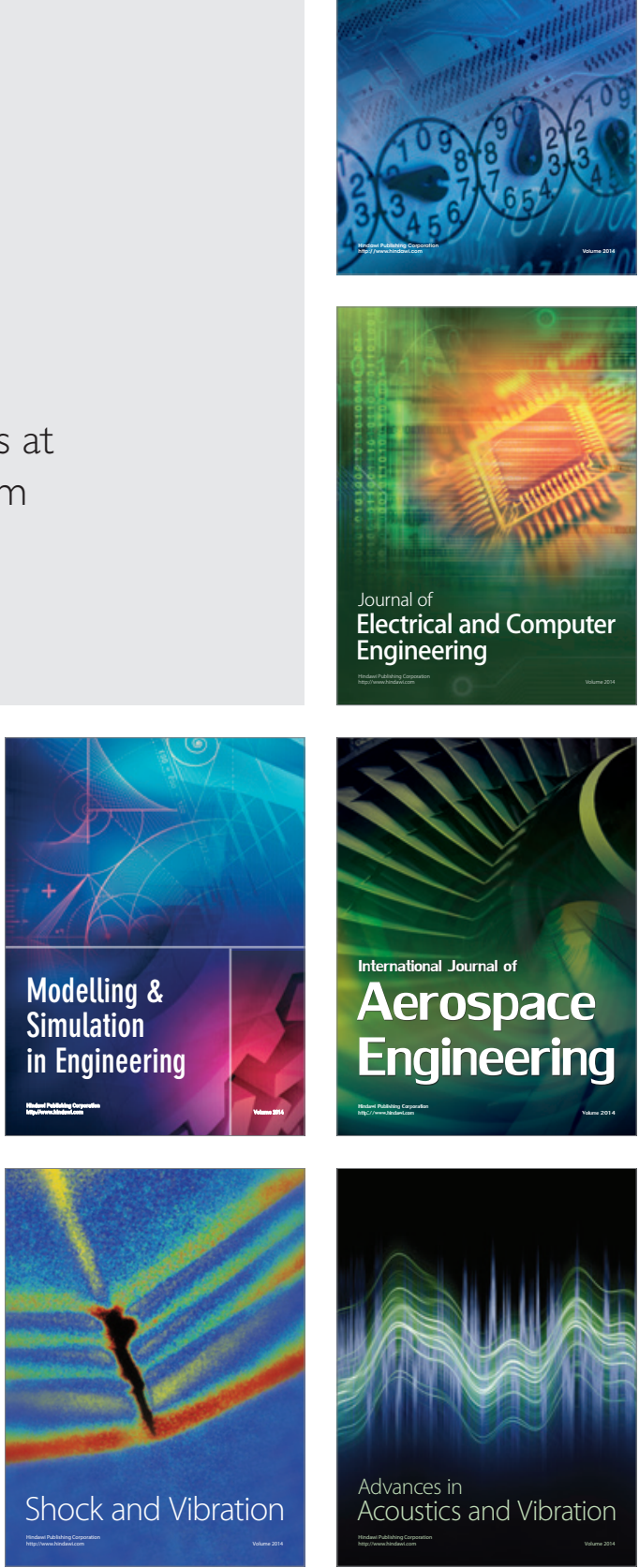